\title{
Diverging Tendencies in Multidimensional Secession
}

\author{
Arne Soulier, Natalie Arkus, and Tim Halpin-Healy \\ Physics Department, Barnard College, Columbia University, NY, 10027-6598, USA
}

Received on 2 May, 2003

\begin{abstract}
We review mean-field and fluctuation-dominated behaviors exhibited by the Seceder Model, which moves an evolving population to various critical states of self-organized segregation, delicately balancing opposed sociological pressures of conformity \& dissent, and giving rise to rich ideological condensation phenomena. The secession exponent and finite societal Seceder limits are examined.
\end{abstract}

This paper discusses recent research [1] on the Seceder Model [2], an intriguing far-from-equilibrium stochastic model of opinion dynamics in which the competing tendencies of conformity and dissent cause an evolving population to fragment, disperse, and coalesce, forming distinct groups characterized by free and frequent interchange of individuals. We imagine a population that is ultimately economic, sociological, or political in character, with interactions based upon shared investment strategies, cultural opinions, or electoral inclinations. This is in sharp contrast to many well-known examples of inanimate cluster formation found in Nature (e.g., in the astrophysical context- Saturnian rings, globular clusters, Virgo galactic supercluster, etc.), where the underlying forces are physical in origin; here, the interactions are based upon signaling, opinion, and information exchange. The Seceder Model bears close spiritual kinship to a number of distinguished evolutionary minority games, among them the Zhang \& Challet variant [3] of Arthur's El Farol Bar problem [4], as well as its stochastic generalization by Johnson and coworkers [5], where it was discovered that the introduction of chance brought the population to a state of self-organized segregation in which two groups adopted diametrically opposed strategies. Further work by Hod \& Nakar [6] revealed a dynamical phase transition in this setting, between 2-group segregation and single-group clustering, driven by the economic cost-benefit ratio implicit in the model. Relevant, too, is the work of Hauert and collaborators [7, 8], who studied 3-group dynamics amidst cooperation, defection, and abstention in a noncompulsory public goods game, mischievously dubbed by popular science pundits [9] as the "Physics of Loners"! In its initial rendering, they considered a well-mixed formulation in which participants could interact readily with any other member of the population. Afterwards, they introduced spatial structure, finding peculiar traveling wave phenomena within the model; see Hauert's web page. Finally, in a paper titled "Meet, Discuss, and Segregate!" Weisbuch et al. [10] consider a model of opinion dynamics in which a population of interacting agents adjust continuous posi- tions via random binary interactions subject to threshold constraints. High thresholds (large inertial barriers) produce a societal convergence of opinion to a single group in overall agreement, whereas low thresholds result in multiple opinion clusters. These, and related consensus models, such as those of Bonabeau [11], Sznajd [12], and others, have been discussed in wry pedagogical fashion by Stauffer in a nice review [13]. Issues on a grander scale were addressed by Stauffer and his close Brazilian colleagues [14].

The Seceder mechanism was devised to demonstrate that a dynamics favoring individuality, yet permitting conformity, cannot only create distinct groups, but also yields a rich diversity of cluster-forming dynamics. The essential trick was to benefit individuals that distinguish themselves from others. This is natural, for one recalls from an epidemological point of view, genetic differences can enhance long-term survival probabilities. Similarly, for players in a minority game or traders on the stock market floor, distinct strategies can yield large returns. The Seceder Model is succintly stated: Within a population of $N$ individuals, each described by a $d$-dimensional opinion vector (representing a multicomponent investment strategy, ideological or political position), we implement the following update algorithm-

- A member (the "voter") of the population is chosen at random to revise his position.

- Pick $m$ individuals (a "polling group") from the population; calculate the mean vector of this subset.

- From among this selection multiplet of size $m$, choose the most distinct member; i.e., the fellow farthest from the mean- that is, the subset center of mass.

- The "voter" adopts an ideological position nearby the dissenter; aside from a superposed, but small, deviate, this adjustment is perfect. [15]

Clearly, the model as defined induces two intrinsically opposed tendencies. If the polled group is tightly-knit in the first place, the result is to enhance homogeneity, since the voter, potentially at some ideological distance from that subset, vacates his position and effectively conforms. On the other hand, should there be an outlier among the selec- 
tion multiplet, the voter choses the underdog, rather than the majority view. Therefore, dissent, and by extension, secession, is implicitly encouraged. Of course, there is a catch, since distinct individuals frequently, but not always, inspire a following, thereby becoming part of the mainstream themselves. In dissenting, they guarantee the demise of their distinction. In what follows, we employ the standard convention that $N$-updates of the population corresponds to one generational time-step. Using this definition, each individual will, on average, be replaced once per generation. Our initial condition is always the same, an entirely homogeneous population with all individuals ideologically located at the origin.

For convenience, we consider first the case $d=1$, a single ideological axis, and investigate the $m$-dependence of this Seceder Model. We expect the limit $m \rightarrow N$, which guarantees enhanced cross-correlation throughout the society, to elicit inevitably, a simpler "mean-field" type of behavior, if only in the extreme case when $m=N$, when we're averaging over the entire population using the societal mean to determine the most distinct, and ultimately, adopted position. In fact, this is what we discovered first, numerically. The shock, though, comes with the suddenness of the transition. There is, surprisingly, a marked change of behavior as we switch from a triplet $(m=3)$ to quartet $(m=4)$ polling groups. In Figure 1a, we show single runs of the Seceder Model using multiplet selection $m=3-8$, within a large population, $N=512$. For $m=3$, we have classic Seceder pattern formation, with self-similar branching characterized by three dominant, but fluctuating arms, with ample smallscale stochastic structure associated with the transient appearance of variously short-lived subbranches. Rather than a gradual crossover, we find that for $m_{c}=4$, the typical stable configuration abruptly involves two groups, not three. In addition, these two branches exhibit only minor fluctuations. Setting $m=5$ further diminishes the fluctuations (one barely notices any subbranches here...), but hardly affects the tilt of what seems to be the nearly linear space-time trajectories of the two divergent groups. Next, for $m=6 \& 7$, there is, strangely, a discrete jump to an altogether different, but closely allied pair of segregating groups. With $m=8(\& 9)$, another jump in velocity is evident in our space-time plot. So it goes with each successive even-odd pair of multiplet selection group. Thus we see that the dominant dynamic of Seceder involves, for $m \geq m_{c}=4$, selforganized segregation into two evenly populated opposing groups with continuous interchange of individuals over the course of time. Similar dynamical segregation was reported by Johnson et al., within their stochastic evolutionary minority game.

Ensemble averaging over many realizations, we can systematically study the growth of the population diameter over time. Figure $1 b$ shows a a double-log plot of that diameter for multiplet selection groups $m=3-12$, drawn from a population $N=512$, with 3000 runs guaranteeing decent statistics. Inferences made previously from the single runs are quickly corroborated here- for $m \geq 4$, we retrieve essentially linear behavior, with successive even/odd selection groups paired up; indeed, the logarithm renders them nearly indistinguishable already at times $t>100$ for $m=6 \& 7$, though they can be separated easily enough at earlier times. The figure renders apparent the decreasing gap size in the discrete velocity spectrum. The scaling exponents are $0.99^{+}$ for $m=8-10$, fitting data for $t \in(1000,3000)$. Only for the case of triplet selection, $m=3$, where the segregative dynamics involves stable multiple-branch solutions, do we see a very different power-law scaling behavior. Indeed, for $m=3$, which corresponds to the lowest curve in Figure $1 \mathrm{~b}$, the diameter asymptotically scales with a secession exponent of $3 / 4$, our extracted value being $0.74 \pm 0.01$.

It's interesting to consider finite-size effects within the extreme societal Seceder limit. In Figure 1c, we show the generic behavior when the entire population serves as the selection group. A glance at this figure reveals that for the smallest populations, $N=m=3-6$, the dominant effect is a single, fairly tightly knit group that is essentially localized near the origin; i.e, the trajectory wanders little from the horizontal time axis. While there is, of course, no branch formation per se for $N=m=3$, we see already for $N=m=4$, the occasional appearance of a short-lived branch budding off of the primary Brownian path. The effect is amplified for $N=m=5 \& 6$ where the transient branching phenomena is more apparent, especially in the latter case where we notice that there is an effectively entropic repulsion between the newly created branch and its ancestor; i.e., they head off at some characteristic angle (in this case, roughly 45 degrees) w.r.t. each other. At these population sizes, there's little difference typically between trajectories generated by triplet and higher multiplet selection dynamics. For $N=m=7 \& 8$, however, we gain a glimpse of the true mean-field asymptotic behavior- a strong tendency to bifurcate into two dominant groups, heading off symmetrically from the origin. Occasionally, branch-splitting occurs; though this happens less and less frequently for increasing $m$, the branches are longer lived. Note, especially the clear progression in this manner for $N=m=6,7,8$. As before, we notice a strong temporal correlation between the statistical death of one branch, marked by the nearly immediate birth, or splitting off of another, so as preserve the sanctity of choice. In this context, were a branch to die, without replacement elsewhere, the result would be a single group (nearly all identical individuals), anathema to a secessional dynamic which encourages difference, at least for a while. For larger population sizes, $N=m=10 \& 25$, we see essentially deterministic divergence of the two groups. Of course, on larger time scales, splitting would inevitably occur for these smallish mesoscopic populations, but the asymptotic behavior is clear. For infinite $m$, the trajectories would be linear, symmetrically splayed about the origin, in a V-pattern, suggested already in Figure 1a. 
$d=1$ Stochastic Seceder Model
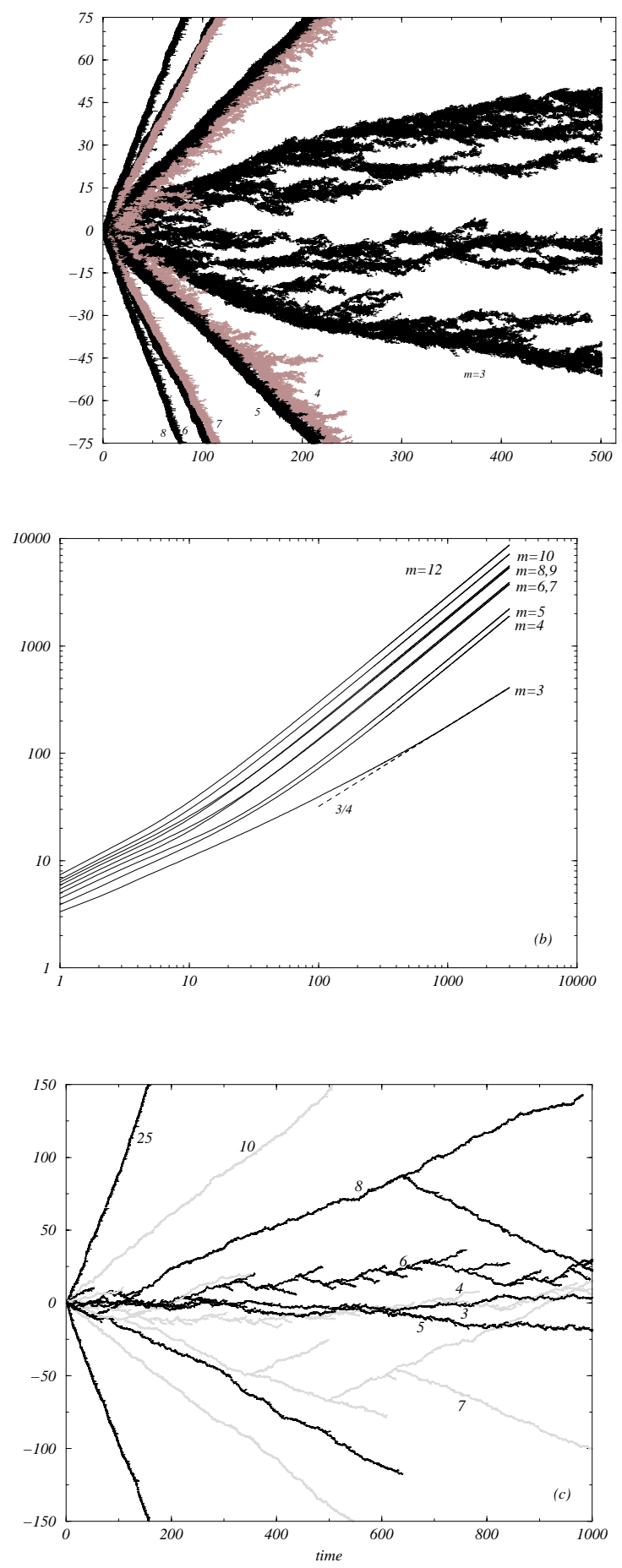

Figure 1. a) Single runs for the 1d stochastic Seceder model, using selection multiplets $m=3,4 \& 5,6 \& 7,8$, within a population of size $N=512$, b) Scaling plot of the divergent population diameter, averaged over 3000 realizations. For $m \geq m_{c}=4$, the behavior is essentially linear, whereas a triplet selection produces a divergent population that scales as $t^{3 / 4}$, and c) Finite-size effect within the societal Seceder model, wherein the entire population serves as the selection group. Shown are the cases $m=N=3-8,10,25$.

Investigating higher dimensions, $d=2$ and 3 , we discover that the critical multiplet selection group size remains fixed at $m_{c}=4$, but that the rate of secession scales nearly linearly in these cases even for $m=3$. More specifically, we show in Figure 2a, the time trace of the three divergent groups in a typical two dimensional simulation. Notice the roughly equilateral arrangement of the three branches. A careful examination of the temporal evolution of the population diameter reveals, see Figure $2 \mathrm{~b}$, that at early times $(t<100)$ the group separation grows as a square root- the solid line in the figure has slope 1/2. By contrast, later on, the separation rate is just a hair sublinear- the dashed line has slope 1. Fits to the final 2000 time steps yields exponents 0.889,0.941,0.992 for population sizes $N=512,1024,2048$, respectively. Simulations in $d=3$ produce very similar results; e.g., the secession exponent being 0.948 for the intermediate population size. Perhaps the most stunning conclusion from the higher dimensional numerics is that additional degrees of freedom associated with extra ideological axes are, from the point of view of the triplet Seceder dynamic, mainly irrelevant since the typical dynamical situation involves collapse to an hyperplane containing just three groups. If one starts, in $d=3$, with four equally-sized parties at the corners of a tetrahedron, it simply won't last- one group always goes extinct! It is an intrinsically unstable configuration, eventually suffering dimensional-reduction to a flat triangular arrangement.

$d=2$ Triplet Seceder Model
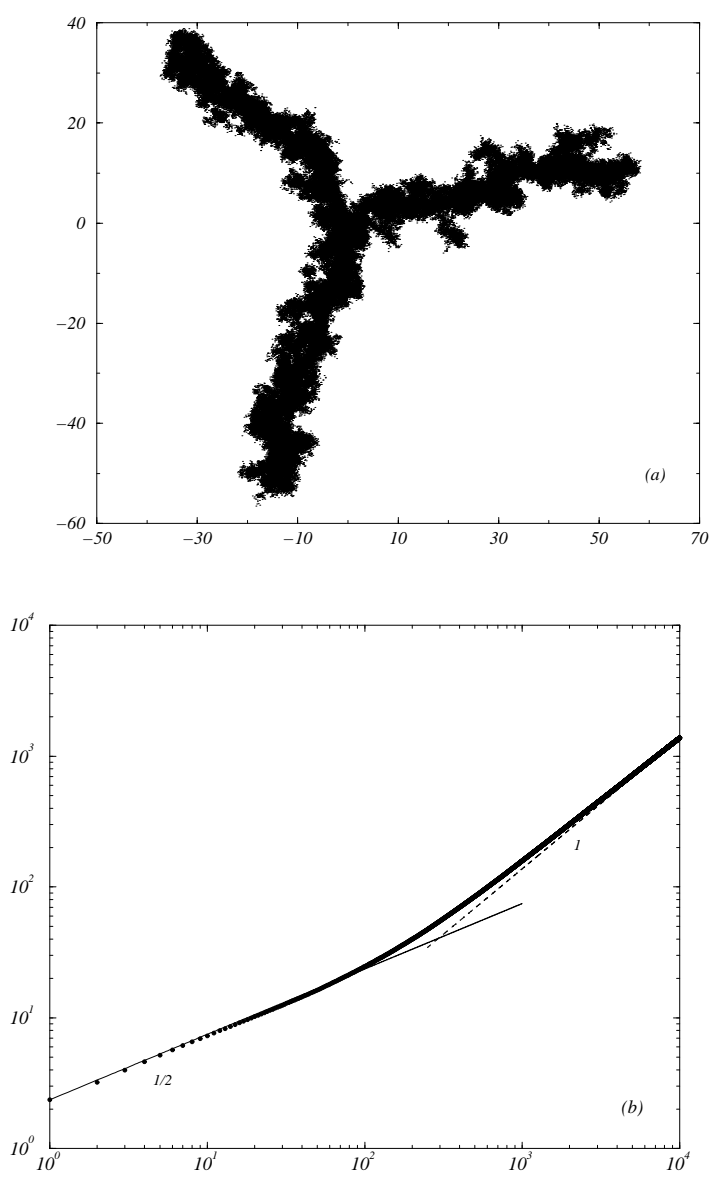

Figure 2. a) Short-time trace in the xy-plane of $2 \mathrm{~d} m=3$ triplet Seceder Model, and b) Scaling plot of the divergent population diameter for the same model, averaged over 1000 realizations. Slopes of the fit lines are indicated. 
Interestingly, much can be gleaned about this stochastic Seceder Model by considering a deterministic, discretized version written as a system of coupled, nonlinear replicator equations, [16] well-known to some members of the mathematical biology/game theory community. For example, if we consider the simplest case of the triplet $(m=3)$ selection dynamics and study the stability of $B=3$ branches (opinion groups), the relevant first-order rate equations read:

$$
\begin{aligned}
& \dot{x}_{1}=x_{1}^{3}+3 x_{1}\left(x_{2}^{2}+x_{3}^{2}\right)+3 x_{1} x_{2} x_{3}-x_{1} \\
& \dot{x}_{2}=x_{2}^{3}+3 x_{2}\left(x_{1}^{2}+x_{3}^{2}\right)-x_{2}
\end{aligned}
$$

where $x_{1,2,3}$ represent population fractions of the three clusters. We've not bothered with the equation for $\dot{x}_{3}$, since it is identical to the first (modulo interchange of indices); probability conservation requires $x_{1}+x_{2}+x_{3}=1$, and since group 2 lies midway between $1 \& 3$ on the 1D ideological axis, it is fundamentally disadvantaged in matters of distinction. If we examine the equation for $\dot{x}_{1}$, the nature of the RHS should be apparent- the cubic term promotes homogeneity [17], the next- secession [18], while the trilinear piece, involving equal representation in the polled group by each of the three branches, is shared equally by the growth variables of the outer two groups [19]. These equations are trivially solved (though less and less so as $B$ increases...), and we find several unstable fixed points- $(1,0,0),\left(\frac{1}{2}, \frac{1}{2}, 0\right)$, and permutations therein, as well as a single superstable fixed point (FP): $\left(\frac{2}{5}, \frac{1}{5}, \frac{2}{5}\right)$. Investigating the situation for $B=4$ groups, we have:

$$
\begin{aligned}
& \dot{x}_{1}=x_{1}^{3}+3 x_{1}\left(x_{2}^{2}+x_{3}^{2}+x_{4}^{2}\right)+3 x_{1} x_{2} x_{3}+6 x_{1} x_{3} x_{4}-x_{1} \\
& \dot{x}_{2}=x_{2}^{3}+3 x_{2}\left(x_{1}^{2}+x_{3}^{2}+x_{4}^{2}\right)+3 x_{2} x_{3} x_{4}-x_{2}
\end{aligned}
$$

Note, again, that all terms are third order, because we're still looking at triplet selection, $m=3$. In any case, because the stable branches are symmetric about its central axis, the flow equations for the remaining variables are easily obtained via the interchange $x_{1} \leftrightarrow x_{4}$ and $x_{2} \leftrightarrow x_{3}$ and, indeed, the globally stable fixed point (no negative eigenvalues!) must lie within this reduced subspace, mirror variables identified. For the case at hand, invoking the constraint $x_{2}=1 / 2-x_{1}$ and demanding $\dot{x}_{1}=0$ leads to the cubic equation $7 x_{1}^{3}-6 x_{1}^{2}+5 / 4 x_{1}=0$, yielding $\left(x_{1}, x_{2}, x_{3}, x_{4}\right)=\left(\frac{5}{14}, \frac{1}{7}, \frac{1}{7}, \frac{5}{14}\right)$, in addition to the 2 branch solutions $\left(\frac{1}{2}, 0,0, \frac{1}{2}\right)$ and $\left(0, \frac{1}{2}, \frac{1}{2}, 0\right)$. Of course, if we brute force numerically integrate the coupled ODEs on the computer, following the trajectories from a randomly generated initial condition, we flow with $100 \%$ probability to this unique superstable fixed point. The situation for $B=5$ recalls the 3 -branch case; try it! You'll find the stable FP $\left(\frac{4}{13}, \frac{2}{13}, \frac{1}{13}, \frac{2}{13}, \frac{4}{13}\right)$, though there's an unstable solution, $\left(\frac{1}{4}, \frac{1}{4}, 0, \frac{1}{4}, \frac{1}{4}\right)$, self-similar to the 2-branch $\left(\frac{1}{2}, 0, \frac{1}{2}\right)$. The 6branch is eaily seen to be irrational, involving solution of the cubic equation $20 x_{2}^{3}+80 x_{2}^{2}+133 x_{2}-17=0$, possessing one real, 2 imaginary roots. Don't bother looking for an 8branch FP; it doesn't exist! Instead the flows will bring you to $\left(\frac{11}{40}, \frac{1}{8}, 0, \frac{1}{10}, \frac{1}{10}, 0, \frac{1}{8}, \frac{11}{40}\right)$, exactly self-similar to the $B=3$ superstable FP $\left(\frac{2}{5}, \frac{1}{5}, \frac{2}{5}\right)$. For additional details regarding the self-similar hierarchical gapped spectrum intrinsic to triplet selection, see [1]. Finally, the replicator formalism provides some hidden extra benefits, among them an explanation for the critical critical selection multiplet size $m_{c}=4$. Assuming arbitrary $m$, one performs a stability analysis of the 2-branch $\mathrm{FP}\left(\frac{1}{2}, 0, \frac{1}{2}\right)$, focussing on the Seceder term arising from the multinomial expansion [1]; in this fashion, we discover $\dot{x}_{2}=\left[2\left(\begin{array}{c}m \\ 1\end{array}\right)\left(\frac{1}{2}\right)^{m-1}-1\right] x_{2}+O\left(x_{2}^{2}\right)$, so we flow back to vanishing $x_{2}$ for $2 m \leq 2^{m-1}$; i.e., $m \geq 4$, as the prefactor of the quadratic term is easily shown to be negative. Lastly, with the replicator equations, we see why the greatest degree of population fragmentation occurs for the case of one ideological dimension where three groups are the norm though 4, 5, 6 groups are not uncommon. By contrast, in $d \geq 3$, the population self-organizes to a coarsened trio of three tightly-knit opinion clusters, effectively collapsing to a 2D ideological plane [1].

Our present efforts focus on the political perspective- understanding the kinetic symmetry breaking phenomena, intermittent dynamics, and subpopulation fluctuations intrinsic to the Seceder mechanism [20].

THH is grateful to P.M.C. \& S. M. de Oliveira for hospitality during his brief visit to UFF-Niteroi (Boa Viagem, Brasil), and to D. Stauffer for advice and a muchremembered introduction to percolation theory. N. Arkus* $\&$ THH were supported by NSF DMR-0083204, Condensed Matter Theory. Finally, many thanks to John Lee for help with a multitude of computational issues.

*NSF-RUI Undergraduate Summer Research Asst.

\section{References}

[1] A. Soulier and T. Halpin-Healy, Phys. Rev. Lett. 90, 258103 (2003).

[2] P. Dittrich, F. Liljeros, A. Soulier and W. Banzhaf, Phys. Rev. Lett. 84, 3205 (2000).

[3] D. Challet and Y.-C. Zhang, Physica A 246, 407 (1997).

[4] W.B. Arthur, Am. Econ. Assoc. Proc. 84, 406 (1994).

[5] N.F. Johnson, P.M. Hui, R. Jonson and T.S. Lo, Phys. Rev. Lett 82, 3360 (1999).

[6] S. Hod and E. Nakar, Phys. Rev. Lett. 88, 238702 (2002).

[7] C. Hauert, S. De Monte, J. Hofbauer, and K. Sigmund, Science 296, 1129 (2002).

[8] G. Szabó and C. Hauert, Phys. Rev. Lett. 89, 118101 (2002).

[9] Phys. Rev. Focus, http://focus.aps.org/v10/st10.html

[10] G.Weisbush, G. Deffuant, F. Amblard, and J.-P. Nadal, Complexity 7, 55 (2002).

[11] E. Bonabeau, G. Theraulaz, and J. L. Deneubourg, Physica A217, 373 (1995).

[12] K. Sznajd-Weron and J. Sznajd, Int. J. Mod. Phys. C 11, 1157 (2000).

[13] D.Stauffer,Sociophysics Simulations, cond-mat/0210213.

[14] S. Moss de Oliveira, P. M. C. de Oliveira, and D. Stauffer, Evolution, Money, War and Computers, (Teubner, Leipzig and Stuttgart, 1999). 
[15] To be more explicit- if $\mathbf{r}$ denotes a position vector in the $d$ dimensional opinion space, then $\mathbf{r}_{\text {voter }}^{\prime}=\mathbf{r}_{\text {dissenter }}+\varepsilon$, where the components of $\varepsilon$ are randomly drawn from the interval $\left\{-\frac{1}{2},-\frac{1}{2}\right\}$. The voter's new ideological position is close to that of the dissenter, but not an exact replica. The two individuals thus become of the same mindset, though their opinions are not identical.

[16] J. Hofbauer and K. Sigmund, Evolutionary Games \& Population Dynamics, (Cambridge University Press, UK, 1998).

[17] Here, like begets like since the polled multiplet, $\{1,1,1\}$, is uniformly represented by group 1 . With $x_{1}$ the probability of a randomly chosen member of the population being in group 1 , the likelihood of a selection triplet being strictly group 1 is just $x_{1}^{3}$.

[18] In this case, a group 1 member is a solitary minority in the polled subset, outnumbered 2-to-1 by either a group 2 (or 3) majority; even so, the underdog that wins the vote. The probability of this happening is $x_{1} x_{2}^{2}$ (or $x_{1} x_{3}^{2}$ ), there being three ways, however, in either scenario.

[19] This presents a democratic dilemma of sorts, since the polling group cannot decide between the two outmost groups, 1 and 3 . In this paper, we simply flip a coin. The combinatoric prefactor, 6 , is thus split in two, equal contributions of $3 x_{1} x_{2} x_{3}$ going to the rates $\dot{x}_{1}$ and $\dot{x}_{3}$. In ref [20], below, the dynamical effects of ideological symmetry breaking, which removes this degeneracy, are considered in some detail, particularly in regard to strong fluctuations within party memberships of the different groups.

[20] A. Soulier and T. Halpin-Healy, cond-mat/0305356; a Seceder applet is available via this abstract. 